Portland State University

PDXScholar

Electrical and Computer Engineering Faculty

Publications and Presentations

2020

Introduction to the Design and Simulation of Reflectionless Filters

\author{
G. Lemire \\ Portland State University, glemire@pdx.edu \\ Branimir Pejcinovic \\ Portland State University, pejcinb@pdx.edu
}

Follow this and additional works at: https://pdxscholar.library.pdx.edu/ece_fac

Part of the Electrical and Computer Engineering Commons Let us know how access to this document benefits you.

Citation Details

Pejcinovic, B., and Lemire, G. "Introduction to the Design and Simulation of Reflectionless Filters," accepted for the 43rd International Convention on Information and Communication Technology, Electronics and Microelectronics (MIPRO), Opatija, Croatia, 2020.

This Conference Proceeding is brought to you for free and open access. It has been accepted for inclusion in Electrical and Computer Engineering Faculty Publications and Presentations by an authorized administrator of PDXScholar. Please contact us if we can make this document more accessible: pdxscholar@pdx.edu. 


\title{
Introduction to the Design and Simulation of Reflectionless Filters
}

\author{
G. Lemire* and B. Pejcinovic* \\ * Portland State University, Portland, OR, USA \\ glemire@pdx.edu, pejcinb@pdx.edu
}

\begin{abstract}
One novel approach to improving filter performance is to use so-called reflectionless filters which have recently been proposed. In this paper we demonstrate how to design, simulate and manufacture a reference reflectionless low-pass filter with the corner frequency of 188 MHz. We have found very good agreement between measurements, simulations, and published data, which validates our implementation of the design procedure. We have also designed a comparable diplexer so that we can examine the relative advantages and disadvantages of the reflectionless filters vs. diplexers. Measurements, simulations and published data, all agree very well in this case as well. This agreement extends into multi-GHz range, far into the filter stopband, which illustrates the high quality of the modeling tools used. The reflectionless filter has been verified to provide low return loss for all ports in the stopband which enables cascading. It also has a much simpler bill-ofmaterials and uses only three unique components, whereas the diplexer uses 32 unique components. On the other hand, the diplexer exhibits steeper initial fall-off after the 3-dB corner frequency and somewhat improved attenuation in the stopband.
\end{abstract}

Keywords - reflectionless filters; filter design; diplexer design; simulation.

\section{INTRODUCTION}

Maximizing useful signal power from the source to the load has been a fundamental design goal in electrical networks since the dawn of modern electronics in the early 20th century [1]-[3]. The mechanism for this matching source to load impedance was usually through some intermediary circuit located between the source and the load. The impedance matching network or more generally "filter" can be as simple as a single termination resistor placed near the load, to an active multitap adaptive broadband filter to quarter wavelength microstrip stubs. Regardless of complexity or target application in which the filter is required, these structures can have one or several design parameters to be optimized such as: maximum power transfer, selective filtering (passband) for a frequency range of interest, and controlling reflection of the signal from the load in an unwanted (stopband) frequency range of interest

Depending on the application and filter topology chosen, in general the design becomes more difficult and complex the more design parameters that need to be optimized. Additionally, many of the current solutions have problems or shortcomings which include inherently limited bandwidth (e.g. quarter wavelength stubs), excessive passband loss, reflective peaking in the transition region and can have a large physical topology which makes implementation difficult for space limited applications. Often the shortcomings of the filter design necessitate additional elements that need to be inserted into the system design to mitigate the unwanted effects.

A subclass of filters called absorptive filters allows for optimization of both passband and stopband characteristics as compared to conventional reflective-type filters. As the name suggests, absorptive filters attenuate the unwanted out of band incident signals by absorption through resistive elements rather than reflection. The concept of absorptive filtering has been around for many decades, with one of the earliest and simplest form of this type of filter is a leaky wall filter which couples unwanted energy to an auxiliary signal path with an absorptive load [1]. No comprehensive list of the different types of absorptive filters was found in the literature, but for the purposes of this work Bulja et al. [4] suggested that absorptive filters can be categorized into transmissive-type absorptive filters and reflective-type absorption filters. Transmission-type absorptive filters can come in several different topologies. The first provides two or more signal paths, of which the sideband path(s) are used to attenuate the stop band signals of the transmission path. The second topology contains the absorptive elements in the body of the filter.

A classical approach to designing a filter that is matched in both the passband and the stopband is to use a diplexer (or multiplexer for more than two frequency bands) and then terminating all but the main path with matched loads. A diplexer is a three-port network that splits the incoming signal from the common port into two frequency dependent signal paths. Diplexers can be realized in admittance complimentary or impedance complimentary architectures [8], [9].

To address the out of band reflective problem a novel methodology for absorptive filters has been recently proposed in [5]-[7]. The authors term these filters reflectionless rather than absorptive because theoretically they have reflection coefficients of zero at all ports for all frequencies for ideal elements [10]. To follow their terminology, these filters will be designated henceforth as reflectionless. This filter has the similar out of band properties as a diplexer but with a simpler design process and more importantly, all ports are matched due to symmetry. This allows for cascading of reflectionless filter sections without the debilitating inter-stage interaction found with many filters. 


\section{REFLECTIONLESS FILTER DESIGN}

The proposed methodology makes use of even-odd mode analysis, symmetry, duality and couples these basic concepts with Cauer ladder network topologies for filters to realize a unique topology for reflectionless filters. Symmetry follows from even-odd mode analysis and this allows the derived filter to have impedance matching at all ports, which implies that the cascading of these structures is at least theoretically possible without interactions between stages.

\section{A. Even-Odd Mode Analysis}

Even-mode excitation is defined as impressing two signals which are equal in both magnitude and phase on each port simultaneously. Odd-mode excitation is defined as impressing two signals equal in magnitude but opposite in phase on each port simultaneously. Even and Odd mode equivalent half circuits are sufficient to completely describe the behavior of a symmetric two-port network.

In Figure 1a a symmetric 2-port network is shown. In Figure $1 \mathrm{~b}$, the network is divided on its symmetry plane and pulled apart exposing wires representing the central nodes of the circuit. First, even-mode excitation is applied to the two ports and because of symmetry no current will flow in any of the exposed wires, thus creating an open circuit which can be represented in Figure 1c. Similarly, when odd mode excitation is applied to the two ports the wires will be at virtual ground and the circuit can be represented in Figure 1d.
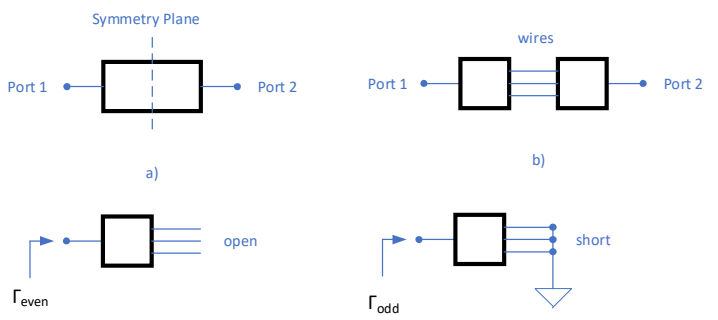

Figure 1. Two port representation of even and odd modes

For the even and odd mode equivalent circuits [5] defines reflection coefficients $\Gamma_{\text {even }}$ and $\Gamma_{\text {odd }}$. For the even-mode circuit

$$
\Gamma_{\text {even }}=S_{11}+S_{12} \text { and by symmetry } \Gamma_{\text {even }}=S_{22}+S_{21}
$$

Similarly, for the odd-mode circuit

$\Gamma_{\text {odd }}=S_{11}-S_{12}$ and by symmetry $\Gamma_{o d d}=S_{22}-S_{21}$

By rearranging and setting $S_{11}=S_{22}=0$ it can be seen that

and

$$
\Gamma_{\text {even }}=-\Gamma_{\text {odd }}
$$

$$
S_{11}=\frac{\mathrm{z}_{\text {even }}-1}{\mathrm{z}_{\text {even }}+1}=-\frac{\mathrm{z}_{\text {odd }}-1}{\mathrm{z}_{\text {odd }}+1}=-\frac{1 / \mathrm{y}_{\text {odd }}-1}{1 / \mathrm{y}_{\text {odd }}+1}=\frac{\mathrm{y}_{\text {odd }}-1}{\mathrm{y}_{\text {odd }}+1}
$$

By inspection it can be seen that

$$
\mathrm{Z}_{\text {even }}=\mathrm{y}_{\text {odd }} \text { and } \mathrm{S}_{21}=\Gamma_{\text {even }}
$$

These two relationships are key to realizing reflectionless filters. A general procedure for realizing a reflectionless filter is presented next. To help illustrate the procedure a reflectionless low-pass filter will be designed using the above two key relationships.

Steps 1 and 2: First, design the even mode equivalent halfcircuit. The left side of the Figure 2 represents the even mode equivalent circuit of a low pass filter using $\mathrm{S}_{21}=\Gamma_{\text {even. }}$. Second, construct the odd mode dual using $\mathrm{Z}_{\text {even }}=\mathrm{y}_{\text {odd, }}$ as shown on the right side of the Figure 2.

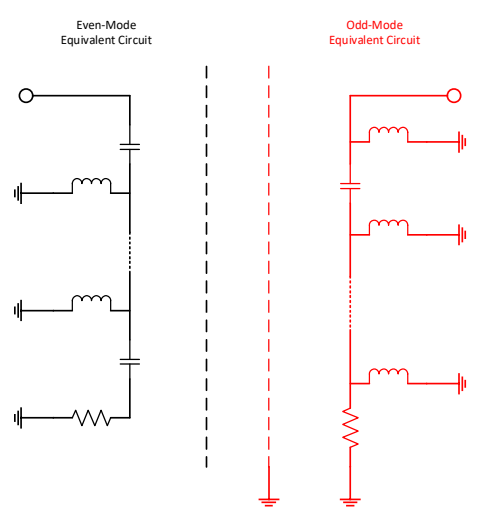

Figure 2. Design of low-pass prototype: even-mode section on the left, and odd-mode section on the right.

Step 3: Symmetrizing the circuit in two steps:

a) redrawing the circuit into more convenient form, as shown in Figure 3. Redrawn components are in red.

b) adding components to make the overall circuit symmetric, as shown in Figure 4. New components are in red. Note that they are superfluous as they do not affect circuit function because they either have a short-circuit in parallel or end in an open-circuit.

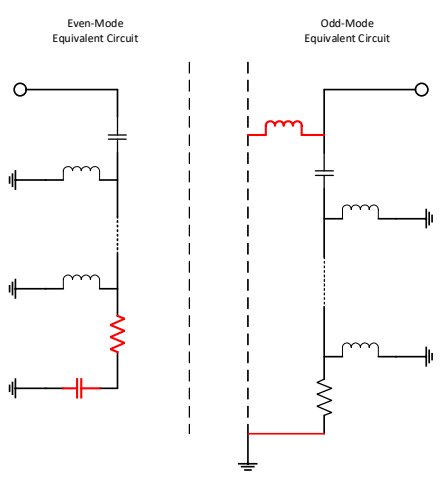

Figure 3. First step in symmetrizing the design: redraw circuit from Figure 2. Redrawn components are in red. 

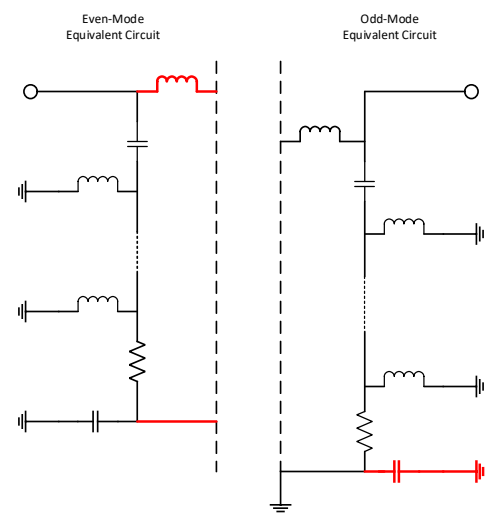

Figure 4. Second step in symmetrizing: adding superfluous components (in red).

Step 4: even and odd sections are connected to form the full filter, as shown in Figure 5.

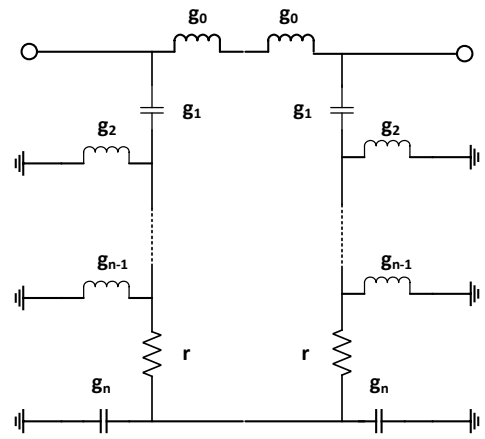

Figure 5. Final low-pass filter prototype of arbitrary order $n$ and normalized component values.

With the topology of the filter established, normalized component values can now be determined, with the values being guided by both symmetry and duality constraints and can be generalized for any characteristic impedance. Duality implies that inductor $\mathrm{g}_{0}$ must be the dual of the capacitor $g_{1}$ (step 2). Determining component values to satisfy the duality condition we have for the first two components

$$
L_{0}=Z_{o}^{2} C_{1}
$$

and substituting the impedance of both inductor and capacitor elements into the above equality gives

$$
\frac{g_{0} Z_{0}}{\omega_{c}}=Z_{o}^{2} \frac{g_{1} Y_{0}}{\omega_{c}}
$$

By inspection $g_{0}=g_{1}$, and similarly, using duality, it can be seen that $g_{1}$ must be the dual of $g_{2}$ and using this same reasoning we can infer $g_{0}=g_{1} \cdots=\cdots g_{k}$ and $\mathrm{r}=1$.

\section{B. Ideal Filter Simulation}

As explained in [5], design of a single filter stage is limited to odd orders and the third order filter stage gives the best compromise among various requirements. To obtain the required characteristics, it is usually necessary to cascade several filter sections, as explained later.
First, we will examine ideal filter behavior using simulation program ADS [12]. Filtering performance of a low-pass filter (LPF) consisting of a cascade of four stages where each stage is of the third order is shown in Figure 6. Magnitude of $\mathrm{S}_{21}$ clearly illustrates where passband and stopband regions are and $3-\mathrm{dB}$ cutoff frequency for this filter is $188 \mathrm{MHz}$. For the magnitude of $\mathrm{S}_{11}$, also known as return loss, we present two plots. One plot is for circuit simulation using elements with four significant digits and the other for using six significant digits. Obviously, the more significant digits we use the closer we are to ideal behavior. However, practical implementations will have much worse tolerances, and therefore fewer significant digits. Practical components are specified between $1 \%$ and $5 \%$ of the nominal value. Note that the return loss for ideal filters is very small across all frequencies.

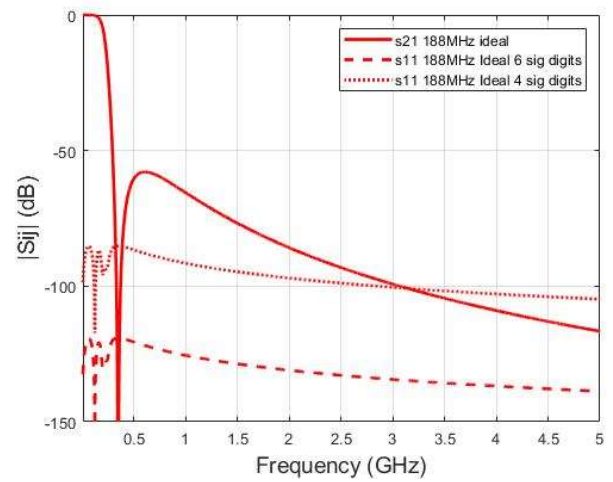

Figure 6. Ideal low-pass filter performance. The amount of return loss is presented for the component accuracy of four and six significant digits.

Next, we will examine reflectionless filters which use non-ideal components using simulations as well as measurements of the manufactured filter.

\section{FILTER IMPLEMENTATION}

The PCB stack-up and material used for this work is well beyond what is required for both the frequency of operation as well as the complexity of the circuit. The specific materials were chosen because the stack-up is familiar, well controlled and PCB panels are regularly run at Synopsys which allows the opportunity to include the test cards in a standard panel. The stack up is 12-layer low-loss Tachyon-100G material and the material properties are shown in Table I [13].

TABLE I. PRINTED CIRCUIT BOARD PROPERTIES, TACHYON-100G

\begin{tabular}{|c|c|c|c|c|}
\hline $\begin{array}{c}\text { No. of } \\
\text { layers }\end{array}$ & $\begin{array}{c}\text { Dielectric } \\
\text { thickness (mils) }\end{array}$ & Dk & Df & $\begin{array}{c}\text { Copper thickness } \\
\text { (mils) }\end{array}$ \\
\hline 12 & 3.25 to 6 & 3.02 & 0.0021 & 0.6 or 2 \\
\hline
\end{tabular}

Obviously, we cannot purchase SMD components with precision of six significant digits, as given in Table II. Instead, we decided to use components within 5\% tolerance with values closest to the ideal ones, as shown in Table II. 
TABLE II. IDEAL AND ACTUAL SMD COMPONENTS SELECTED FOR FILTER IMPLEMENATION. COMPONENT TOLERANCES ARE 5\%.

\begin{tabular}{|l|l|l|l|}
\hline & $\boldsymbol{L}(\mathbf{n H})$ & $\boldsymbol{C}(\boldsymbol{p F})$ & $\boldsymbol{R}$ (Ohms) \\
\hline Ideal & 22.7176 & 9.08706 & 50 \\
\hline Non-ideal (5\%) & 23 & 9.1 & 49.9 \\
\hline
\end{tabular}

A four-stage filter, equivalent to a 12-th order traditional filter, was designed and implemented in this technology. Photo of the final PCB is shown in Figure 7. PCB has dimensions of 3" $\mathrm{x} 1$ ".

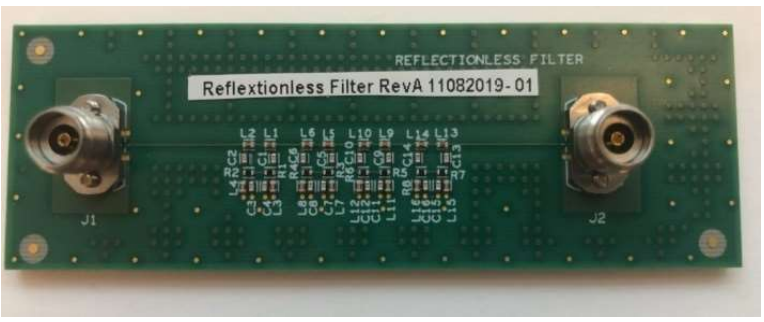

Figure 7. PCB implementation of reflectionless LPF with four cascaded sections and equivalent filter order $\mathrm{n}=12$ with corner frequency 188 $\mathrm{MHz}$.

\section{A. Simulated and Measured LPF Results}

Before the LPF was manufactured, we examined its performance relative to the ideal case by using ADS simulation. Results are shown in Figure 8 which demonstrates that $\mathrm{S}_{21}$ behaves almost ideally up to $1.5 \mathrm{GHz}$. After that frequency we suspect that some interactions among parasitics in components and layout cause significant differences. Return loss $\left(\mathrm{S}_{11}\right)$ is very far from ideal but it is less than $20 \mathrm{~dB}$ up to $1.5 \mathrm{GHz}$. This would indicate that $S_{11}$ is more sensitive than $S_{21}$ to departures from ideal component values.

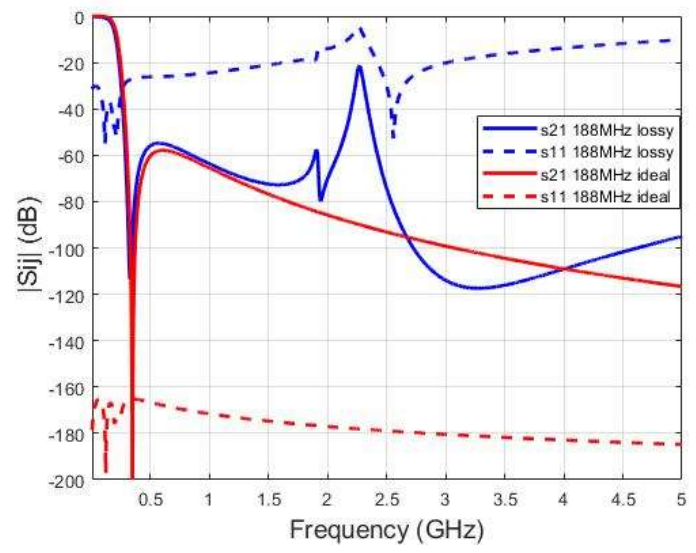

Figure 8. Simulated $S_{11}$ and $S_{21}$ for reflectionless filter with ideal and realistic (lossy) components.

Final test of any design is a comparison between simulation and measurements. The circuit shown in Figure 7 was carefully measured on a $40 \mathrm{GHz}$ VNA. Effects of the fixture were removed by de-embedding using AFR (Automatic Fixture Removal). As shown in Figure 9, we observed excellent agreement between simulation and measurements. We attribute this to very accurate component models [11] and careful circuit simulation which includes most external parasitics, such as transmission line effects. This gives us confidence that more challenging designs, e.g. those using higher frequencies, and circuit optimization will also be successfully simulated and manufactured.

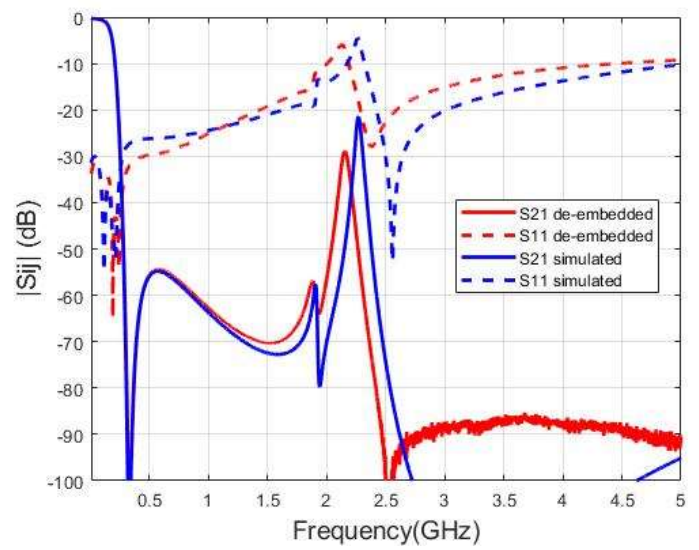

Figure 9. Simulated and measured $\mathrm{S}_{11}$ and $\mathrm{S}_{21}$ for the reflectionless LPF

\section{DIPLEXER DESIGN AND IMPLEMENTATION}

To evaluate the quality of the reflectionless filters return loss, a conventional filter with similar low reflection out of band qualities was chosen to be used as a comparison. A dual diplexer formulation [8], [9] is considered where one constructs low pass and high pass filters that have complimentary input admittances. The filters are connected in parallel at a common port with the input admittance of the diplexer given as $Y_{o}=Y_{p}+Y_{s}$ where $Y_{p}$ and $Y_{s}$ represent the input admittances of the low-pass and highpass filters respectively. A matched filter requires that the input admittance $Y_{o}$ be real and constant for all frequencies on the common port. Filter pairs that satisfy this condition are termed complimentary. The physical implementation of the diplexer in Figure 10 shows the three-port diplexer on the left with the separate two port de-embedding structure on the right. The diplexer common port is on the far left with the low-pass port on the upper center and the high-pass port on the lower center. For this work, and following [6], an order $\mathrm{n}=16$ Butterworth diplexer was chosen as a comparison.

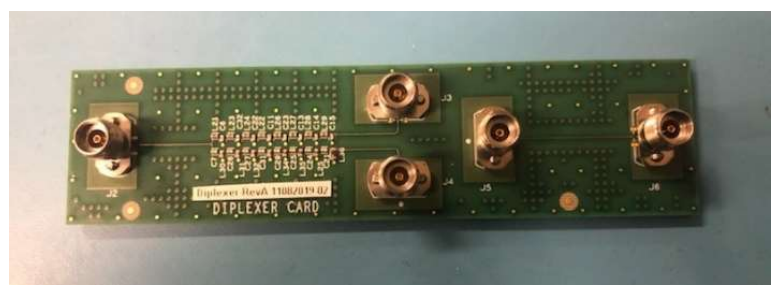

Figure 10. Diplexer implementation of LPF on the left. Structure on the right is used for deembedding.

The comparison of the reflectionless and the diplexer LPFs are shown in Figure 11. The reflectionless LPF displays similar responses for $S_{21}$ except the transition 
region is initially less steep. More importantly, the $\mathrm{S}_{11}$ response for reflectionless LPF is superior to the diplexer across almost the entire frequency range.

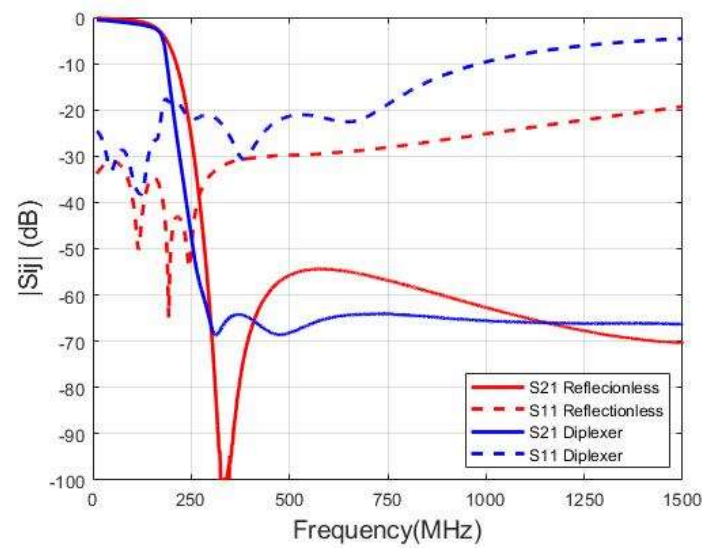

Figure 11. Comparison of measured $\mathrm{S}_{11}$ and $\mathrm{S}_{21}$ for reflectionless and diplexer implementation of LPF.

Another important feature of the reflectionless filter is the symmetry property. The filter is a two-port device and because of symmetry the return loss at both ports is theoretically identical. This allows these filters to be cascaded with other filters or with other sensitive 50-ohm components with little interaction. Figure 12 demonstrates that the reflectionless filter has very close to identical return loss responses $S_{11}$ and $S_{22}$. The diplexer is matched for $S_{11}$ (common port) relatively well but $\mathrm{S}_{22}$ shows almost unity reflection in the stop band. Any noise above the passband frequency on diplexer port 2 will tend to reflect from the filter rather than being absorbed with possible resonance ramifications between the filter and any connected circuits.

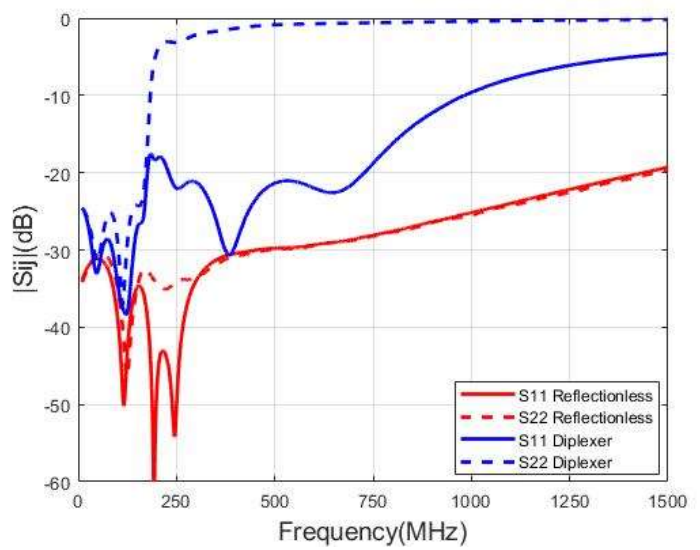

Figure 12. Comparison of measured return loss on both ports of reflectionless and diplexer implementation of LPF.

\section{CONCLUSION}

We have demonstrated and verified the novel reflectionless filter design procedure outlined in Morgan and Boyd's papers. A low pass reflectionless filter prototype was realized. A classical Butterworth diplexer prototype satisfying the same requirements was also realized and used to evaluate the quality of the return loss characteristics for the reflectionless filter. The prototype measurement results obtained for reflectionless filter and equivalent diplexer show that the reflectionless filter has improved return loss characteristics over a wide frequency range. We have also observed very good matching between simulation and measured response for reflectionless filters. Return loss of both ports on reflectionless filter was superior to diplexer design. Diplexer has an advantage of steeper fall-off in the stopband albeit in a limited frequency range close to the corner frequency.

\section{ACKNOWLEDGMENT}

We would like to thank Synopsys for use of their lab and for manufacturing circuits, Keysight for providing ADS simulation software, and to Modelithics for providing SMD component models for ADS.

\section{REFERENCES}

[1] V. Met, "Absorptive Filters for Microwave Harmonic Power", Proc, IRE, vol. 47, no. 10, pp. 1762-1769, Oct. 1959. DOI 11.1109/JRPROC.1959.287111

[2] H.W. Bode, "Network Analysis and Feedback Amplifier Design" New York: D. Van Nostrand Company, 1945.

[3] G. Matthaei, L. Young, and E. Jones, "Microwave Filters, Impedance Matching Networks, and Coupling Structures". Norwood, MA: Artech House, 1980

[4] S. Bulja, A. Grebennikov, and P. Rulikowski, "Theory, analysis and design of high order reflective, absorptive filters", IET Microwaves, Antennas and Propagation, March 2017. DOI 10.1049/ietmap.2016.0431

[5] M. A. Morgan, "Think Outside the Band: Design and Miniaturization of Absorptive Filters," in IEEE Microwave Magazine, vol. 19, no. 7, pp. 54-62, Nov.-Dec. 2018. DOI: 10.1109/MMM.2018.2862541

[6] M.A. Morgan and T.A. Boyd, "Technical and experimental study of a new class of reflectionless filter", IEEE Trans. Microwave Theory and Techniques, vol. 59, no. 5, pp 1214-1221, May 2011. DOI 10.1109/TMTT.2011.2113189

[7] M. A. Morgan and T. A. Boyd, "Reflectionless Filter Structures," IEEE Transactions on Microwave Theory and Techniques, vol. 63, no. 4, pp. 1263-1271, Apr. 2015, doi: 10.1109/TMTT.2015.2403841.

[8] G .L. Matthaei and E. G. Cristal, "Theory and Design of Diplexers and Multiplexers", Advances in Microwaves, vol. 2, pp 237-326, 1967.

[9] R. G. Veltrop and R. B. Wilds, "Modified Tables for the Design of Optimum Diplexers", Electronic Defense Laboratories, Technical Memorandum, No EDL-M559, 3 July 1963

[10] M. A. Morgan, "Reflectionless Filters", Norwood MA: Artech House, 2017

[11] Modelithics Exemplar Library [online] Available: https://www.modelithics.com/

[12] Advanced Design System (ADS), Keysight, [online], Available: https://www.keysight.com/en/pd-2998595/pathwave-advanceddesign-system-ads-2020?cc=US\&lc=eng

[13] Isola group, Tachyon-100G data sheet, [online], Available: $\mathrm{http} / /$ www.isola-group.com/wp-content/uploads/Tachyon-100GLaminate-and-Prepreg-Data-Sheet-0417.pdf 